\title{
Incidental Finding of a Left Atrial Myxoma while Characterising an Autoimmune Disease
}

\author{
Rafael Garcia-Carretero ${ }^{1}{ }^{*}$, Gema Naranjo-Mansillaํ, Esther Luna-Heredia ${ }^{1}$, \\ Paloma Arias-Baldo², Blanca-Nieves Beamonte-Vela ${ }^{1}$ \\ ${ }^{1}$ Department of Internal Medicine, Mostoles University Hospital, Spain \\ 2 Department of Radiology, Mostoles University Hospital, Spain
}

\begin{abstract}
Although cardiac tumours are uncommon, cardiac myxomas account for more than fifty percent of all cases and are the most frequent primary cardiac tumour. They have a broad clinical spectrum, usually related to cardiac symptoms, peripheral embolic events or systemic manifestations. We present a case report of a 68 -year-old man who presented with systemic symptoms and analytical features suggestive of an autoimmune disease. In the ensuing diagnostic procedures, a cardiac myxoma was found, and after surgical resection, both the systemic manifestations and the analytical abnormalities disappeared.
\end{abstract}

Keywords: left atrial myxoma, cardiac benign tumor, constitutional symptoms

Received: 15 March 2018 / Accepted: 26 April 2018

\section{BACKGROUND}

One of the challenges faced by physicians is to deal with undifferentiated and systemic diseases, such as systemic inflammatory response, anorexia, weight loss and fatigue observed in certain conditions, mainly malignancy and autoimmune diseases. This cluster of symptoms is non-specific and can often be misleading. The differential diagnosis includes cardiac myxoma, an uncommon cause of this cluster of symptoms. However, among a variety of clinical manifestations, systemic inflammatory symptoms can account for up to $30 \%$ of clinical features, thus mimicking malignancy or autoimmune-related problems. These unusual symptoms may complicate the diagnostic procedure.

\section{CASe Presentation}

A 68-year-old man was admitted to hospital following the diagnosis of non-iron deficiency anaemia by his general practitioner. The patient reported fatigue, with a feeling of intense weakness. He also stated a weight loss of approximately $8 \mathrm{~kg}$ over the last year and lowgrade fever. In the previous few months, he had expe- rienced episodes of Raynaud syndrome triggered by low temperatures. He had COPD and type 2 diabetes mellitus, and was taking tiotropium bromide and glibenclamide, prescribed by his general practitioner. On physical examination, the only finding was a soft systolic murmur. Otherwise, the investigations revealed no untoward medical issues.

On admission, the patient had a white cell count of $6.1 \times 10^{3} / \mathrm{uL}$, haemoglobin of $11 \mathrm{~g} / \mathrm{dL}$, and a platelet count of $310 \times 10^{3} / \mathrm{uL}$. Kidney and liver function were normal, and his INR was 0.9. However, the erythrocyte sedimentation rate (ESR) was $99 \mathrm{~mm} /$ hour, and C-reactive protein (CRP) was $99 \mathrm{mg} / \mathrm{L}$ Antinuclear antibodies (ANAs) were positive, and indirect fluorescent antibody (IFA) was 1:160 with a homogenous pattern. Anti-SSA (Ro), anti-SSB (La), anti-dsDNA and anticentromere antibodies, and ENA panel were all negative.

\section{Investigations}

The positive ANA test result, the high levels of CRP and ESR, the low-grade fever, the weakness and the persistent fatigue were suggestive of an autoimmune disease. Indeed, the anaemia could be explained as being 
chronic-disease related. However, the patient did not have muscle pain, rash, arthritis or skin sensitivity to light. Analytical findings were subtle and non-specific, making it difficult to establish the diagnosis of an autoimmune disease.

Given the wasting syndrome, the constitutional symptoms and the malaise, it was decided to perform an abdominal ultrasound, and a chest CT scan, to rule out an occult neoplasm. The ultrasound results were normal; however, the CT scan revealed a large left atrial mass that passed through the mitral valve into the left ventricle. Its radiological features could not rule out a left atrial tumour, though the first choice was a large free-floating atrial thrombus (Figure 1). He was referred, therefore, to the intensive care unit (ICU) for close monitoring.

Transthoracic echocardiography showed an $8.8 \mathrm{x}$ $3.5-\mathrm{cm}$ floating mass, which originated from the left atrial ceiling. It passed through the mitral valve and reached the centre of the left ventricle. The systolic function was normal, with an ejection fraction of $65 \%$, but there was moderate mitral stenosis (Figure 2). Further trans-esophageal echocardiography confirmed the findings mentioned above: the mass originated from the interatrial septum, near the drainage of the right superior pulmonary vein.

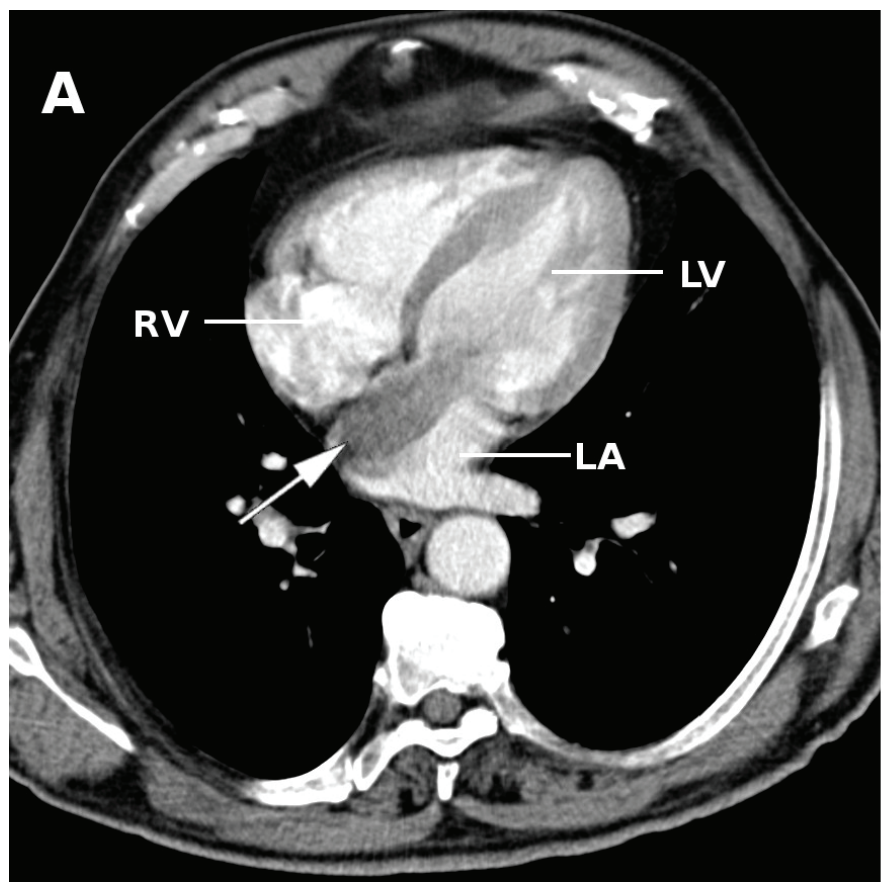

\section{Treatment and follow-up}

The patient was referred to our Cardiac Surgery Department to undergo surgical resection of the left atrial mass within 72 hours of the diagnosis being made. It turned out to be a sizeable reddish mass with fibroelastic consistency. Histopathology revealed eosinophilic spindle cells on a myxoid background and multiple small vessels. The mass stained positive with calretinin.

The post-operative period was uneventful. The patient did not develop severe, respiratory or infectious complications. He recovered, was discharged, and resume normal activity. He had periodic follow-ups to monitor his symptoms and to detect possible complications. After an eighteen-month monitoring period, the patient had gained weight, and the fatigue and weakness had disappeared. Laboratory test abnormalities had improved, the ESR and CRP were normal at 15 $\mathrm{mm} /$ hour and $7 \mathrm{mg} / \mathrm{L}$, respectively, and his haemoglobin level was $14.7 \mathrm{~g} / \mathrm{dL}$. The patient had experienced no further episodes of Raynaud syndrome, which we considered as primary since it did not occur in association with other autoimmune diseases.

\section{Discussion}

The three most common differential diagnoses, regarding cardiac masses, are large vegetations, intracardiac

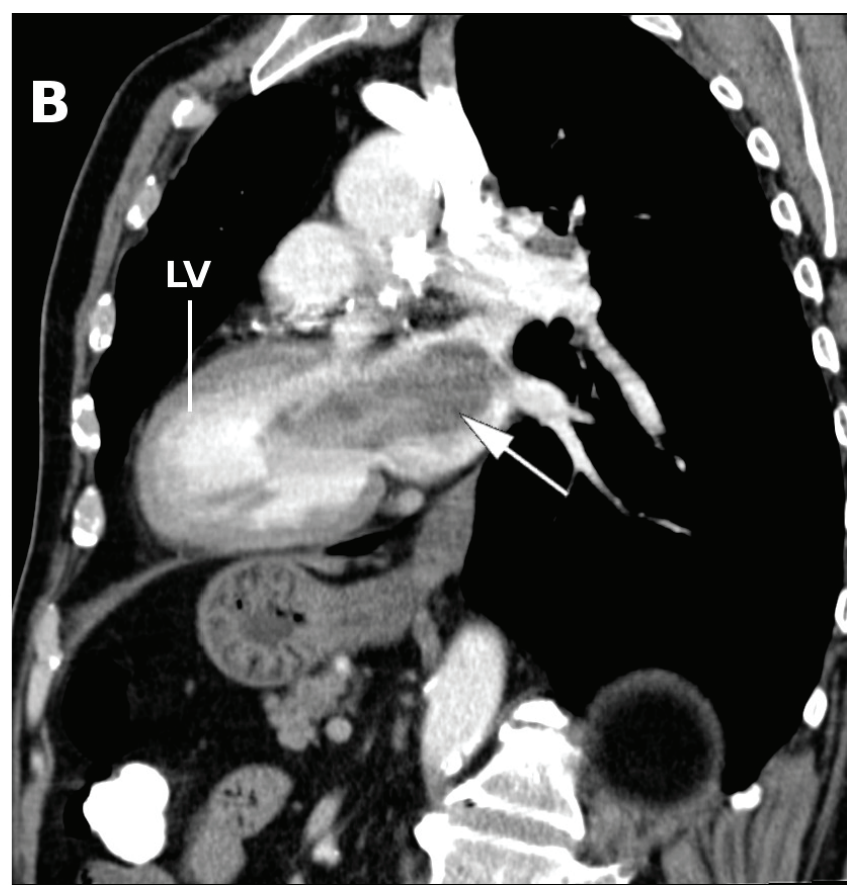

Fig. 1. The CT scan with intravenous contrast medium administration showed a large left atrial mass that passed through the mitral valve into the left ventricle, in both slides: horizontal plane (A) and sagittal plane (B). Radiologists could not rule out an intracardiac thrombus (big white arrow). RV: right ventricle. LV: left ventricle. LA: left auricle. 

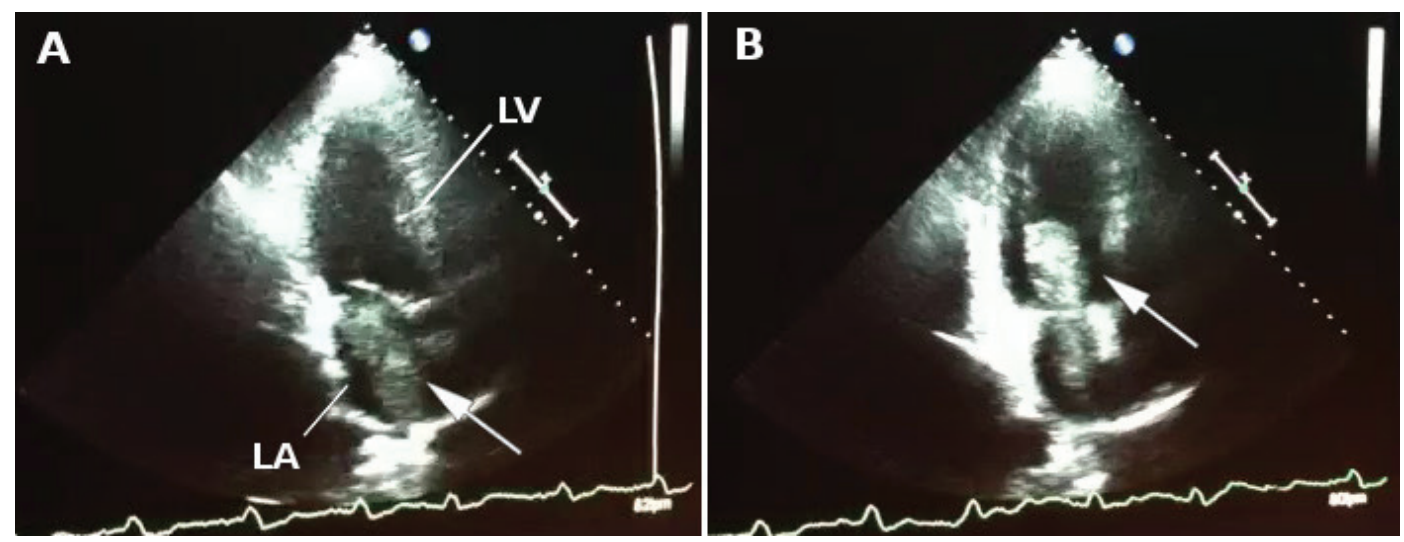

Fig. 2. Echocardiography revealed a floating mass (white arrow), which originated from the interatrial septum, near the drainage of the right superior pulmonary vein. Slide A (systole) shows the mass within the left auricle (LA). However, the mass prolapsed through the mitral valve and reached the centre of the left ventricle (LV) in diastole (slide B).

thrombi and tumours, and although echocardiography plays an essential role in detecting these masses, identifying their nature is difficult. Cardiac tumours represent $0.2 \%$ of all human tumours and can be primary or metastatic. Primary tumours are uncommon, representing less than $0.1 \%$, and about $50-75 \%$ are benign tumours, i.e., cardiac myxomas.[1]

Intracardiac masses can have a broad clinical spectrum. Cardiac symptoms such as fatigue, exertional dyspnoea, valve dysfunction or arrhythmias can be present. Peripheral and cerebral embolic events, or systemic manifestations such as low-grade fever, weight loss and weakness, are also included in the cluster of symptoms.[2]

The patient's symptoms, although subtle and nonspecific, were suggestive of a systemic inflammatory disorder, often seen in malignancy and autoimmune diseases. The early analytical findings were in line with the likelihood of an autoimmune disease. However, he did not have any skin, muscle or joint manifestation. Since a connective tissue disease diagnosis was not definite, we decided to perform a full-body CT scan which would aid in the detection of malignancy as a potential cause of his symptoms. The main finding was an unexpected intracardiac mass, which later turned out to be a left atrial myxoma.

Cardiac myxomas are uncommon tumours. They can originate in any cardiac chamber, even in valves, but they are usually found as a pedunculated mass arising from the left atrium, near the fossa ovalis.[1] The extensive clinical spectrum which is presented in association with these cases can make the diagnosis complicated, and it can only be established by the clinical team systematically considering every possibility.
Many case-series studies have identified heart failure, peripheral or central embolisms as the main spectrum of clinically significant complications due to atrial myxomas.[2,3] Some recently published case reports have highlighted unusual or uncommon symptoms, such as arrhythmias, [4] fever of unknown origin,[5] brachial artery embolism,[6] subclavian artery embolism,[7] central retinal artery occlusion[8] and multiple cerebral infarctions.[9] These acute presentations contrast with systemic symptoms such as malaise and weight loss, as reported in recent papers.[10,11] The clinical signs and symptoms found in our patient, as well as the analytical features, led us to consider either an autoimmune disease or an occult neoplasm. Left atrial myxoma was an unexpected finding that could explain not only the manifestations but also the analytical abnormalities.

We would like to clarify a point regarding the patient's ANA result as we believe it was misleading. Positive ANAs must be interpreted in the context of other laboratory studies and clinical features, and a single positive ANAs need not, on its own, mean that the patient has an autoimmune disease. A dilution of 1:160 could be considered a mild positive result, and could be the result of another condition, such as leukaemia or septic arthritis. Furthermore, a low ANA titre should be ignored in patients with no systemic disease or other manifestations of autoimmune disease.[12] It is worth noting the study by Mendoza et al. [13] in which interleukin-6 (IL-6) was overproduced by cardiac myxomas, and this may have a significant role in the presence of constitutional symptoms and immunologic abnormalities observed in patients presenting with cardiac myxomas. More recently, Kiechl[14] reported the case of a 
Available online at: www.jccm.ro

patient with a 3-year history of an unclassified rheumatic disease and elevated IL-6, who suffered multiple ischemic strokes, and whose final diagnosis was a left atrial myxoma. Finally, Pinede et al. [2] emphasised the importance of the classical presentation of constitutional symptoms of fever, and weight loss, suggestive of a connective tissue disease, due to IL- 6 production. Thus, constitutional signs may be used for monitoring these cardiac tumours. These symptoms usually disappear after removal of the cardiac myxoma, although prolonged elevation of ANA has been noted.[15]

\section{Learning points}

- Although uncommon, atrial myxomas should be considered in the differential diagnosis of an atrial mass. However, in the first instance consideration should be given to the presence of a large thrombus or metastatic tumours. In the absence of further complications such as emboli, arrhythmia, etc., atrial myxomas have an excellent prognosis if promptly treated. Sometimes a diagnosis is difficult to achieve if the symptoms are paucisymptomatic. Non-specific, constitutional symptoms may be misleading and make the diagnosis difficult.

- The final diagnosis of autoimmune disease should be established only after exhaustive diagnostic procedures in patients with highly suggestive signs and symptoms.

- The role of IL-6 and its involvement in the presence of constitutional symptoms resembling an autoimmune disease should be noted.

\section{CONFLICT OF INTEREST DISCLOSURE}

The author declares that there is no conflict of interest regarding the publication of this paper.

\section{REFERENCES}

1. Reynen K. Cardiac myxomas. N Engl J Med. 1995;333:1610-7.

2. Pinede $L$, Duhaut $P$, Loire R. Clinical presentation of left atrial cardiac myxoma. A series of 112 consecutive cases. Medicine
The Journal of Critical Care Medicine 2018;4(2) • 67

(Baltimore). 2001;80:159-72.

3. Lee VH, Connolly HM, Brown RDJ. Central nervous system manifestations of cardiac myxoma. Arch Neurol. 2007;64:111520.

4. Rajani AR, Muaz RN, Govindaswamy PR, Mian MH. Arrhythmias are not to blame for all cardiac syncope patients: left atrial myxoma causing syncope in a middle-aged man. BMJ Case Rep. 2015;2015. doi: 10.1136/bcr-2014-209119.

5. Gavrielatos G, Letsas KP, Pappas LK, Dedeilias P, Sioras E, Kardaras F. Large left atrial myxoma presented as fever of unknown origin: a challenging diagnosis and a review of the literature. Cardiovasc Pathol. 2007;16:365-7.

6. Gururani K, Kumar P. Unusual presentation of left atrial myxoma. BMJ Case Rep. 2016;2016. doi:10.1136/bcr-2016-217089.

7. Kandoria A, Negi P, Mahajan K, Puri S. Left atrial myxoma complicated by acute embolism to the left subclavian artery. BMJ Case Rep. 2016;2016. doi: 10.1136/bcr-2016-215345.

8. Kim J-H, Youn $\mathrm{H}-\mathrm{J}$, Jung $\mathrm{M}-\mathrm{H}$, Oh $\mathrm{C}-\mathrm{Y}, \mathrm{Ahn} \mathrm{S}-\mathrm{H}$, Cho W-H, et al. Retinal artery occlusion by left atrial myxoma misdiagnosed as thrombus. Springerplus. 2016;5:352.

9. Kebede $S$, Edmunds $E$, Raybould A. A large left atrial myxoma causing multiple cerebral infarcts. BMJ Case Rep. 2013;2013. doi: 10.1136/bcr-2013-010007.

10. Dobson R, Tweddle E, Scott M, Cubukcu A. Large left atrial myxoma with an unusual origin. BMJ Case Rep. 2012;2012. doi: 10.1136/bcr-2012-006782.

11. Garcia-Carretero R, Vela BB, Martínez-Quesada G, San Jose Montano B. Demographic and clinical features of atrial myxomas: A case series analysis. Acute Card Care. 2016;18:6569.

12. Malleson PN, Mackinnon MJ, Sailer-Hoeck $\mathrm{M}$, Spencer $\mathrm{CH}$. Review for the generalist: The antinuclear antibody test in children - When to use it and what to do with a positive titer. Pediatr Rheumatol Online J. 2010;8:27.

13. Mendoza CE, Rosado MF, Bernal L. The role of interleukin-6 in cases of cardiac myxoma. Clinical features, immunologic abnormalities, and a possible role in recurrence. Texas Hear Inst J. 2001;28(1):3-7.

14. Boehme C, Zangerle A, Mayer L, et al. Excess Interleukin-6 Production and Multiple Ischemic Strokes - What is the Link? Austin J Clin Neurol. 2017;4:1115

15. Wang HT, Tsai CY, Chang HN, Shih CM, Yu CL. Prolonged elevation of antinuclear antibodies in a patient with atrial myxoma after tumor extirpation. Vol. 13, Clinical and experimental rheumatology. Italy; 1995. p. 676-7. 